\title{
Thermal conductivity measurements of semi-transparent single-walled carbon nanotube films by a bolometric technique
}

\author{
Mikhail E. Itkis, Ferenc Borondics, ${ }^{\dagger}$ Aiping Yu, Robert C. Haddon* \\ Center for Nanoscale Science and Engineering \\ Departments of Chemistry and Chemical \& Environmental Engineering \\ University of California, Riverside, California 92521-0403
}

\section{Supporting Information}

\section{Temperature distribution along suspended SWNT film}

To determine the temperature distribution along the SWNT film we first consider the IR light as a heat generator of zero width illuminating NT film at position $x=L / 2$ (Fig.S1; the effect of a finite width of the illuminated spot is considered later). In vacuum the absorbed heat propagates from the illuminated central region along the suspended SWNT film towards the electrical contacts without losses. In this case steady-state 1D heat flow is described by the equation $d^{2} T / d x^{2}=0$ in both halves of the sample $0<x<L / 2$ and $L / 2<x<L$, with boundary conditions $T=T_{0}$ at $x=0$ and $x=L$, and $d T / d x=P_{a b s} / 2 \kappa A$ at $x=(L / 2)^{-}, d T / d x=-P_{a b s} / 2 \kappa A$ at $x=(L / 2)^{+}$, where $P_{a b s}$ is the absorbed power of IR radiation (which is divided into the left and right heat flows, Fig.1a ), $\kappa$ is thermal conductivity of SWNT film, and $A$ is the cross-sectional area of the SWNT film. The solution gives a triangular temperature profile $T(x)=T_{0}+\left(P_{a b s} / 2 \kappa A\right)^{\star} x$ in the left half of the sample $(0<x<L / 2)$ and $T(x)=T_{0}+\left(P_{a b s} / 2 \kappa A\right)^{*}(L-x)$ in the right half of the sample $(L / 2<x<\mathrm{L})$. This leads to the maximum temperature $T_{M}=\left(P_{a b s} L / 4 \kappa A\right)$ in the middle of the sample and average temperature increase $\Delta T=\left(T_{M^{-}} T_{0}\right) / 2=$ $\left(P_{a b s} L / 8 \kappa A\right)$, which gives Eq.1 for determining the thermal conductivity of the SWNT film in our experimental geometry:

$$
\kappa=\left(P_{a b s} / \Delta T\right)(L / 8 A)
$$




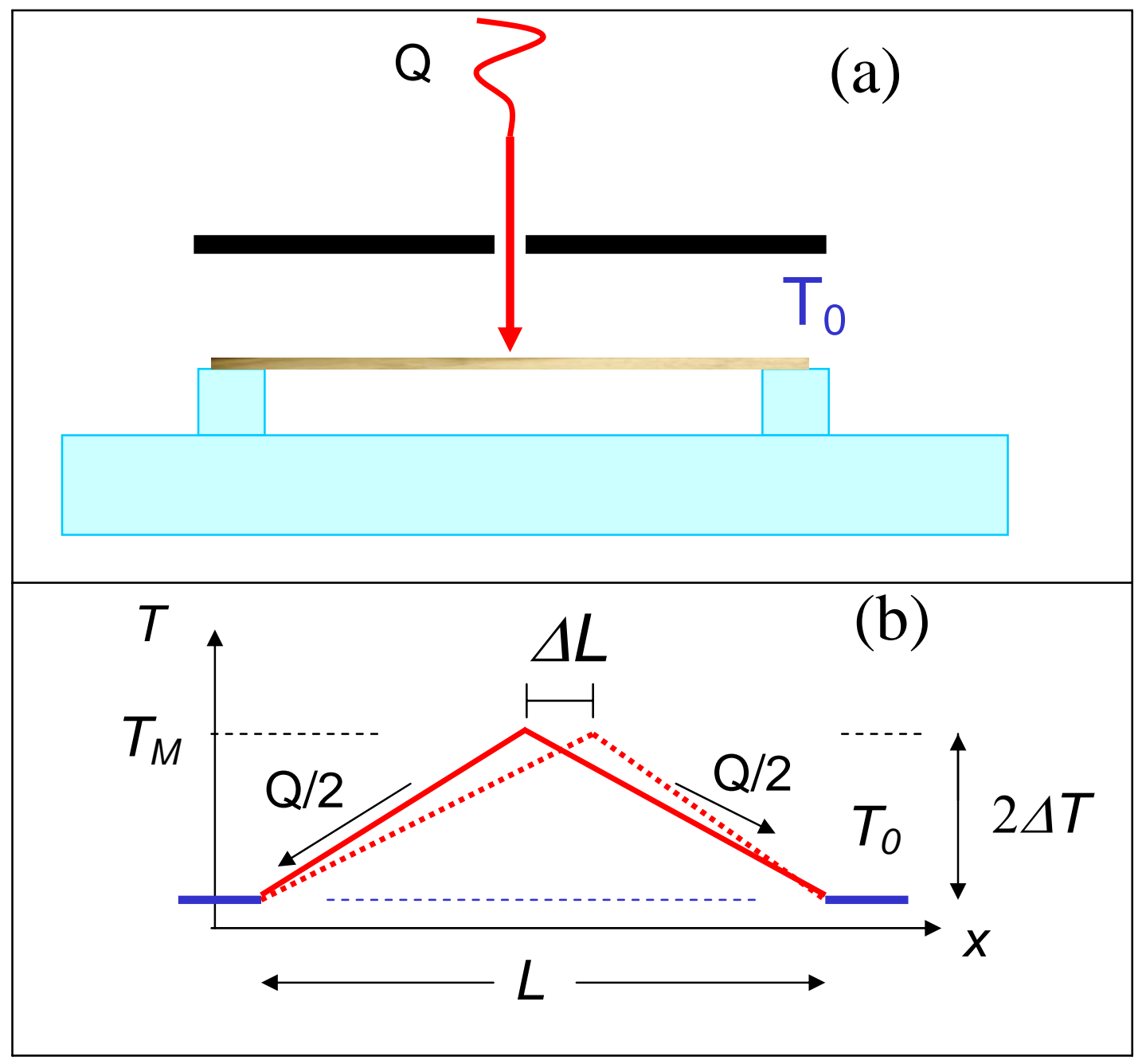

Fig. S1.(a) Experimental set-up for thermal conductivity measurements of thin SWNT film suspended between electrical contacts in vacuum. IR light is illuminating the center of the SWNT film. (b) Triangular temperature profile along the SWNT film in case of in center illumination (red solid line) and off center illumination (red dotted line).

We can estimate the errors in using Eq.1 in the two most likely cases: i) off center position of IR illumination, and ii) finite width of illuminated spot. An off center location of the IR beam (displaced by $\Delta L$ ) leads to uneven heat flow to the left and right current contacts. Adding to the analysis above a condition that the sum of heat flows to the left and to the right is constant, we found that the average temperature increase $\Delta T$ can be expressed as a function of $\Delta L$ in a form: $\Delta T=\left(P_{a b s} L / 8 \kappa A\right)\left[1-(\Delta L / L)^{2}\right]$. Utilizing Eq.1 in this case would lead to a 
measured $\kappa$ value $\kappa_{\text {meas }}$ which is higher than the intrinsic $\kappa$-value, such that: $\kappa_{\text {meas }}=\kappa\left[1+(\Delta L / L)^{2}\right]$. Because of quadratic correction by the $\Delta L / L$ term an off center shift by $\Delta L / L=10 \%$ will lead to only $1 \%$ overestimation of the measured value of $\kappa$.

An IR source of finite width can be represented as a superposition of point sources evenly distributed from $-\Delta \mathrm{L}$ to $+\Delta \mathrm{L}$ in off center positions; integration over these sources would bring an error in determination of $\kappa$ using Eq.1 below $0.25 \%$. In our experimental situation $2 \Delta L=0.4 \mathrm{~mm}$ and $L=3.5-4 \mathrm{~mm}$, thus $\Delta L / L$ is below $10 \%$.

\section{Comparison of the thermal and electrical transport in SWNT films and SWNT/Epoxy composites.}

In a related work, ${ }^{1}$ we studied the performance of AP- and P-SWNT SWNTS epoxy composites as a function of the SWNT loading using steady state heat flow technique, and in Fig. S2 we present an analysis of the transport properties of the dilute SWNT networks formed inside the polymer matrix (thermal conductivity of a neat epoxy is $0.2 \mathrm{~W} / \mathrm{mK}$ ). In comparison with SWNT films transport is further suppressed in the SWNT/epoxy composites: by about 2 orders of magnitude in the thermal conductivity (Fig.3a and S2a), and up to 6 orders of magnitude in the electrical conductivity (Fig.3b and S2b). Note that this suppression is observed well above the electrical percolation threshold. ${ }^{2,3}$

The reduced volume fraction in the SWNT/epoxy composite (10\% in composites in comparison with $100 \%$ in the case of SWNT thin films) and a portion of nonpercolating SWNT branches are only partially responsible for the observed suppression. The other cause for the suppression of the thermal and electrical transport in the transition from a pure SWNT film to the SWNT/epoxy composite is more likely the presence of a very thin $(\sim 1 \mathrm{~nm})$ layer of polymer separating the SWNTs at the intertube junctions which dominates the intertube junction 
resistance. ${ }^{4,5}$ This interfacial layer acts as an insulating barrier which introduces phonon scattering and significantly suppresses electron tunneling.

Comparison of Fig.3c and Fig.S2 shows an interesting trend: the relationship between the Lorenz numbers corresponding to P-SWNTs and AP-SWNTs is inverted under the two sets of experimental conditions. In the case of films, APSWNTs show higher Lorenz numbers than the P-SWNTs (Fig.3c) while for the polymer matrix the Lorenz number is larger for P-SWNTs (Fig.S2c).

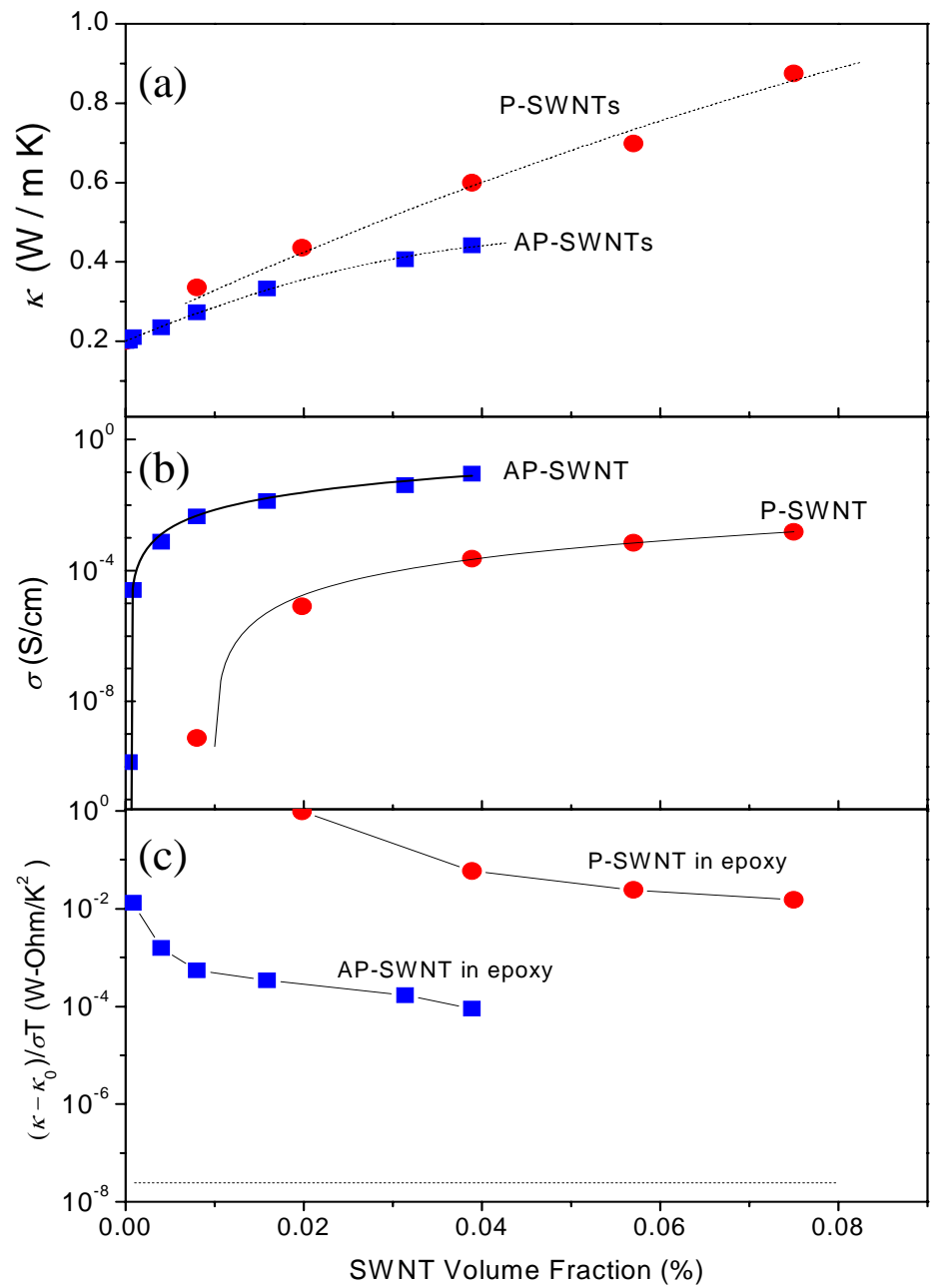

Figure S2. Transport properties of SWNT/epoxy composites composed of purified (P) and as-prepared (AP) SWNTs as a function of SWNT loading: a) Thermal conductivity; b) Electrical conductivity; c) Lorenz numbers. $\kappa_{0}$ corresponds to thermal conductivity of neat epoxy $\kappa_{0}=0.2 \mathrm{~W} / \mathrm{m} \mathrm{K}$ 
Thus thermal and electrical properties of SWNT network are affected in different ways depending on the processing of SWNTs, the degree of dispersion and the resulting nature of intertube junctions. ${ }^{1}$

\section{References}

1. Yu, A.; Itkis, M. E.; Bekyarova, E.; Haddon, R. C. Appl. Phys. Lett. 2006, 89, 133102.

2. Hu, L.; Hecht, D. S.; Gruner, G. Nano Lett 2004, 4, 2513-2517.

3. Bekyarova, E.; Itkis, M. E.; Cabrera, N.; Zhao, B.; Yu, A.; Gao, J.; Haddon, R. C. J. Am. Chem. Soc. 2005, 127, 5990-5995.

4. Shenogin, S.; Xue, L. P.; Ozisik, R.; Keblinski, P.; Cahill, D. G. J. Appl. Phys. 2004, 95, 8136-8144.

5. Shenogina, N.; Shenogin, S.; Xue, L.; Keblinski, P. Appl. Phys. Lett. 2005, 87, 133106. 